\title{
Radiation-hydrodynamics simulations of surface convection in low-mass stars: connections to stellar structure and asteroseismology
}

\author{
Hans-G. Ludwig ${ }^{1,2}$, Elisabetta Caffau ${ }^{1}$ and A. Kučinskas ${ }^{3}$ \\ ${ }^{1}$ Observatoire de Paris-Meudon, GEPI, 92195 Meudon Cedex, France, \\ email: [Hans.Ludwig,Elisabetta.Caffau]@obspm.fr \\ ${ }^{2}$ CIFIST Marie Curie Excellence Team, Observatoire de Paris-Meudo \\ ${ }^{3}$ Institute of Theoretical Physics and Astronomy, Goštauto 12, Vilnius 01108, Lithuania \\ email: ak@itpa.lt
}

\begin{abstract}
Radiation-hydrodynamical simulations of surface convection in low-mass stars can be exploited to derive estimates of i) the efficiency of the convective energy transport in the stellar surface layers; ii) the convection-related photometric micro-variability. We comment on the universality of the mixing-length parameter, and point out potential pitfalls in the process of its calibration which may be in part responsible for the contradictory findings about its variability across the Hertzsprung-Russell digramme. We further comment on the modelling of the photometric micro-variability in HD 49933 - one of the first main COROT targets.
\end{abstract}

Keywords. convection, hydrodynamics, stars: atmospheres, stars: evolution, stars: oscillations

\section{Introduction}

Radiation-hydrodynamical (RHD) simulations of surface convection in low-mass stars have reached a high level of maturity. Such simulations provide quantitative predictions about the spatial and temporal statistics of the flows taking place in the stellar surface layers. We used the 3D RHD code $\mathrm{CO}^{5}$ BOLD to study convective flows in late-type stars at different metalicity. This contribution deals with two distinct applications of $\mathrm{CO}^{5} \mathrm{BOLD}$ models. The first one is related to the efficiency of the convective energy transport in convective envelopes, the second one to the low-level photometric variability related temporal evolution of the surface granulation pattern. We shall rather discuss problems than solutions with a focus on dwarfs.

\section{Convective energy transport in the envelopes of late type-dwarfs}

It is well known from the theory of stellar structure that convection is generally an efficient means of transporting energy, and it establishes a thermal structure close to adiabatic. Only in vicinity of the boundaries of convective regions noticeable deviations from adiabaticity occur. In convective envelopes of late-type stars the upper boundary of the convective envelope - usually located close to or even in the optically thin layers - constitutes the bottle-neck for the energy transport through the stellar envelope assigning a special role to it. Despite it is geometrically thin and contains little mass it largely determines the properties of the convective envelope as a whole. It is the value of the entropy of the adiabatically stratified bulk of the convective $s_{\text {env }}$ which is most important from the point of view of stellar structure since it influences the resulting radius and effective 
temperature of a stellar model. $\mathrm{s}_{\mathrm{env}}$ is controlled by the efficiency of convective and radiative energy transport in the thin, superadiabatically stratified surface layers. Detailed RHD simulations can be applied to model this region allowing to quantify the mutual efficiency of the convective and radiative energy transport and predict senv . Comparing the RHD predictions to standard 1D models based on mixing-length theory (MLT) the value of $\mathrm{s}_{\mathrm{env}}$ can be translated into a corresponding mixing-length parameter $\alpha_{\mathrm{MLT}}$ (see Ludwig et al. 1999 for details).

In stellar evolution calculations the free mixing-length parameter is usually calibrated against the Sun. However, it is unclear whether mixing-length theory provides a suitable scaling of the convective efficiency at constant $\alpha_{\mathrm{MLT}}$ across the Hertzsprung-Russell diagram (HRD), and a lot of work was invested to address this issue empirically. Unfortunately, hitherto, no coherent picture emerged. Here we report on an update of earlier work on the theoretical calibration of $\alpha_{\mathrm{MLT}}$ based on RHD simulations. While not at all comprehensive it illustrates some of the pitfalls in the process which may in part be responsible of the blurred and sometimes even contradictory picture which emerged so far concerning the variability of the mixing-length parameter.

\subsection{Comments on the functional dependence of $\alpha_{\mathrm{MLT}}$ on stellar parameters}

Discussions which took place during the symposium led the authors to add a comment about the question which stellar properties govern the value of the mixing-length parameter $\alpha_{\mathrm{MLT}}$. We argued above that RHD models of the surface layers are able to provide information about $\mathrm{s}_{\mathrm{env}}$ and $\alpha_{\mathrm{MLT}}$. The models are characterised by the atmospheric parameters and consequently the functional dependence of $\alpha_{\mathrm{MLT}}$ can be described in terms of them. Whether the standard atmospheric parameters $T_{\text {eff }}$ and $\log g$ together with the chemical composition are the most suitable coordinates is not clear. One might speculate that, e.g., the surface opacity is a physically more relevant quantity. Nevertheless, for unevolved late-type stars the conditions at the stellar surface govern the global envelope structure and the standard atmospheric parameters are suitable coordinates to parameterise them. Global stellar parameters (mass, radius, or age) play merely an implicit role. The situation only changes when the size of the granular cells or the thickness of the superadiabatic layer become comparable to the stellar radius which might happen in giants. To our opinion one should usually avoid to express changes of $\alpha_{\mathrm{MLT}}$ in terms of, e.g., stellar mass or age since it tends to obscure the underlying physics.

\section{2. $\alpha_{\text {MLT }}$ from six 3D RHD models}

Squares in Figs. 1 and 2 mark the parameter combinations in the $T_{\text {eff }}-\log g$-plane of six 3D RHD models we used for calibrating $\alpha_{\mathrm{MLT}}$. All atmospheres belong to dwarfs, three of solar metalicity, three of a metalicity of $1 / 100$ solar. Our new 3D results are superimposed on earlier results obtained from a grid of 2D RHD models by Ludwig et al. (1999) and Freytag et al. (1999). There are obvious differences of up to $\approx 0.8$ between the $3 \mathrm{D}$ and $2 \mathrm{D}$ calibrated values. Does this indicate that a theoretical calibration with the help of RHD models is hopelessly inaccurate?

The first question to answer is what is a big and what is a small difference when it comes to $\alpha_{\mathrm{MLT}}$. At present interferometry and analysis of eclipsing binaries provide stellar radii to an accuracy of about $1 \%$. Looking at stellar structure models (see, e.g., Lebreton et al. 2001, their Fig. 10) for late-type main-sequence stars the highest sensitivity of the stellar radius and effective temperature to changes of $\alpha_{\mathrm{MLT}}$ is found at about one solar mass. Christensen-Dalsgaard (1997) finds for the Sun a sensitivity of $\delta \ln R \approx-0.24 \delta \ln \alpha_{\mathrm{MLT}}$. Hence, considering the present observationally achievable accuracies we would like to 


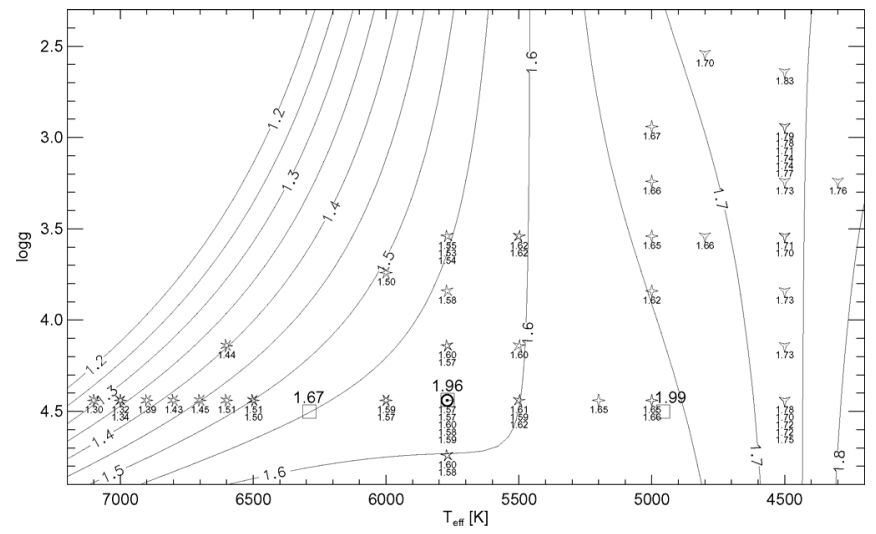

Figure 1. Theoretically calibrated mixing-length parameters at solar metalicity in the $T_{\text {eff }}-\log g$-plane. Squares mark our new results based on 3D models, the other symbols mark earlier 2D results. The obtained values are given by the numbers, the isolines represent a smooth fit to the $2 \mathrm{D}$ data.

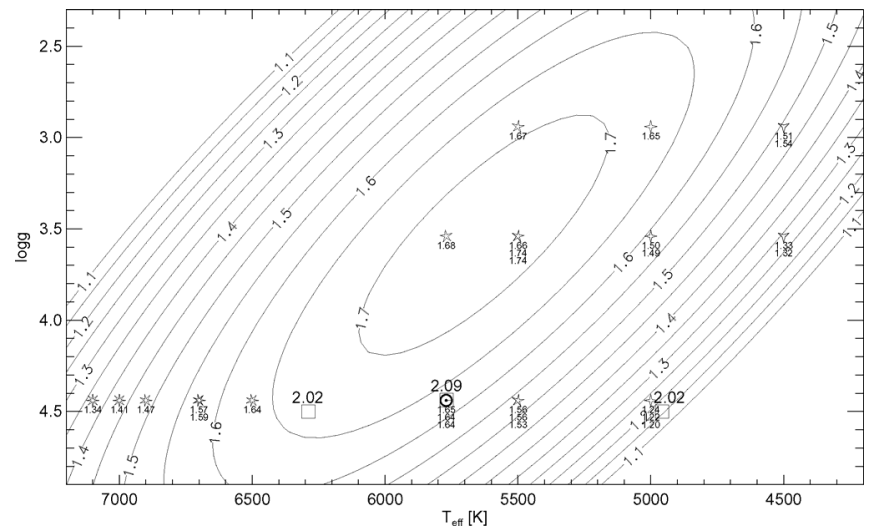

Figure 2. Same as Fig. 1 but for $1 / 100$ of the solar metalicity.

know $\alpha_{\text {MLT }}$ to better than $4 \%$, and the differences between the $2 \mathrm{D}$ and $3 \mathrm{D}$ results are clearly relevant.

We did not mention yet the methodological changes we introduced when calibrating our 3D-based $\alpha_{\mathrm{MLT}}$ 's in comparison to the earlier works: i) Our new values were calculated with a different mixing-length dialect. The earlier results assumed the formulation given by Böhm-Vitense (1958) while we now used the formulation given by Mihalas (1978). ii) Following the general trend in stellar evolution theory, our 1D comparison models are now full-fledged stellar atmosphere models instead of integrating a prescribed $T(\tau)$-relation to describe the atmospheric temperature run as was done in the earlier results. As we shall see in a moment this is a crucial point, in particular for the cool, metal-poor model at $5000 \mathrm{~K}$. iii) It is now well established that the convective energy transport operates more efficiently in $3 \mathrm{D}$ than in restricting $2 \mathrm{D}$ symmetry. Hence, we would expect a systematic bias towards higher mixing-length parameters in $3 \mathrm{D}$ relative to $2 \mathrm{D}$.

Having in mind that we expect larger $\alpha_{\mathrm{MLT}}$-values in $3 \mathrm{D}$ we think that the absolute differences are perhaps not surprising and now rather focus on differential trends with $T_{\text {eff }}$. The statistical uncertainties in the $2 \mathrm{D}$ calibration amount to \pm 0.05 . Taking together the different flavours of MLT, and the different $1 \mathrm{D}$ comparison models used in the 2D 


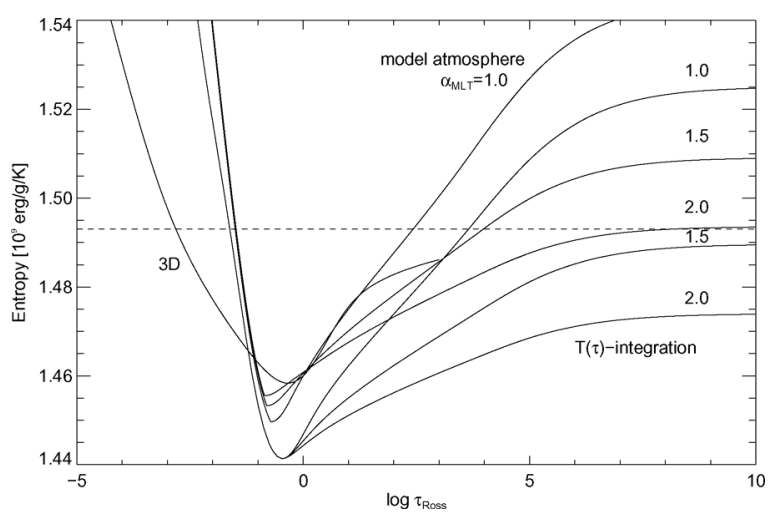

Figure 3. Entropy profiles for 1D stellar atmosphere and stellar structure models for various mixing-length parameters in comparison to the mean 3D stratification for the metal-poor model at $T_{\text {eff }}=5000 \mathrm{~K}, \log g=4.5$, and $[\mathrm{M} / \mathrm{H}]=-2.0$. All $1 \mathrm{D}$ models employ the MLT formulation by Mihalas (1978). The dashed line depicts the entropy senv predicted by the 3D model for the adiabatically stratified part of the convective envelope.

and $3 \mathrm{D}$ calibrations the trends start to look similar in $2 \mathrm{D}$ and $3 \mathrm{D}$ with the exception of the coolest metal-poor model which is far off. What is the reason?

Figure 3 shows a comparison between entropy profiles of 1D MLT based models and of the horizontally and time-wised (on surfaces of equal optical Rosseland depth) averaged $3 \mathrm{D}$ model. This kind of comparison provides the calibrated $\alpha_{\mathrm{MLT}}$. It is obvious that the result depends on the employed 1D models. Using stellar atmosphere models produces a mixing-length parameter which is almost by 0.5 larger than one based on models which use a prescribed $T(\tau)$-relation in the convectively stable part of the stellar atmosphere. The reason is to a large extend the difference in the entropy minimum $s_{\text {min }}$ attained in the deep atmospheric layer in combination with the small overall entropy jump - the difference $s_{\text {env }}-s_{\min }-$ in the star which amounts to only $\approx 1 / 6$ of the solar value. Stars of higher $T_{\text {eff }}$ show larger entropy jumps which makes the exact level of the entropy minimum less critical. The quite different differential behaviour of $\alpha_{\mathrm{MLT}}$ in 3D relative to $2 \mathrm{D}$ is mainly a result of the different choice of comparison model.

While one might consider this as mere technicality we rather believe that part of the confusion about trends and absolute values of the mixing-length parameter have their origin - besides observational problems - in different and not clearly specified procedures of how the MLT-related quantities are computed and the atmospheric structure integration are actually performed in models. It is, e.g., still quite common to use simple grey $T(\tau)$-relations for describing the atmospheric temperature run. Figure 3 illustrates that the particular choice might generate mismatches corresponding to substantial changes in the value of the mixing-length parameter necessary to restore the actual scaling of the envelope entropy with changing stellar effective temperature.

Finally, Fig. 3 illustrates that even full-fledged model atmospheres are not always able to reproduce the entropy minimum in 3D models closely. The reason is that for metalpoor dwarfs $3 \mathrm{D}$ models predict large deviations from radiative equilibrium conditions in the formally convectively stable part of the atmosphere (in the figure apparent by the low entropy of the 3D model at low optical depth). In 1D stellar atmospheresradiative equilibrium conditions are assumed to fix the atmospheric temperatures. Trampedach (2007) puts forward the idea to extract the mean $T(\tau)$-relation from 3D models for performing the atmospheric structure integration. This obviously provides a better match to the 3D atmospheric entropy minimum, and hence a "cleaner" calibration of the 


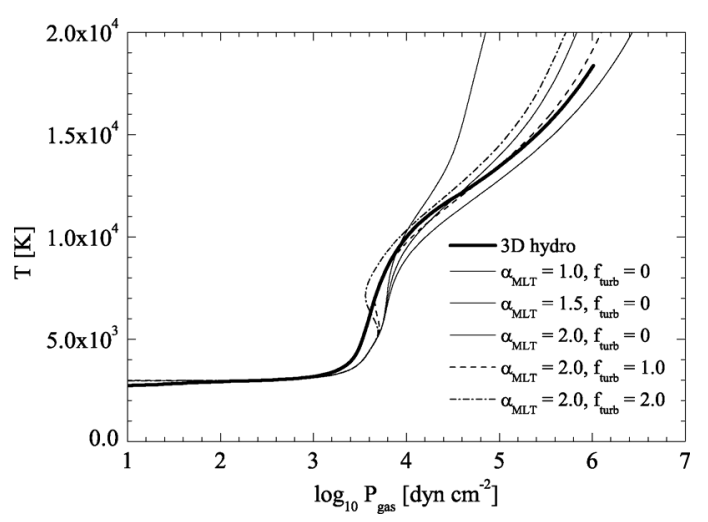

Figure 4. Temperature-pressure profiles of a 3D red giant model (thick solid line) in comparison to $1 \mathrm{D}$ stellar atmosphere models of different $\alpha_{\mathrm{MLT}}$ leaving out (thin solid lines) or including (dashed and dashed-dotted line) turbulent pressure (for details see text).

mixing-length parameter, however, also is more difficult to implement in existing stellar evolution codes. The 3D calibrated values of $\alpha_{\mathrm{MLT}}$ by Trampedach (for solar metalicity and MLT formulation by Böhm-Vitense) are somewhat lower than our values presented here but show a similar trend with $T_{\text {eff }}$ on the main-sequence.

\subsection{Turbulent pressure trouble}

In the main-sequence models discussed above turbulent pressure plays generally only a small role but becomes relatively more important towards lower gravities - and causes extra trouble when one is interested in a well-defined calibration of the mixing-length parameter. Figure 4 shows the average temperature profile of a 3D red giant model $\left(T_{\text {eff }}=3600 \mathrm{~K}, \log g=1.0,[\mathrm{M} / \mathrm{H}]=0.0\right)$ in comparison to standard $1 \mathrm{D}$ model atmospheres of the same atmospheric parameters. While turbulent pressure $P_{\text {turb }}$ is naturally included in the RHD simulation it is modelled in a ad-hoc fashion in $1 \mathrm{D}$ models assuming a paramterisation $P_{\text {turb }}=f_{\text {turb }} \rho v_{\mathrm{c}}^{2}$, where $f_{\text {turb }}$ is a free parameter of order unity, $\rho$ the mass density and $v_{\mathrm{c}}$ the convective velocity according MLT.

Figure 4 shows that in the 3D models the turbulent pressure "lifts" the temperaturegas pressure profile towards lower pressures which is essentially impossible to reproduce in the 1D models - irrespective of the choice of $\alpha_{\mathrm{MLT}}$ and $f_{\text {turb }}$. The failure is related to the local nature of MLT confining the action of the turbulent pressure gradients strictly to the convectively unstable regions. While formally one can still match the thermal profile of the 3D model in the deeper layers by 1D profile with suitably chosen $\alpha_{\mathrm{MLT}}$ and/or $f_{\text {turb }}$ such a match becomes physically little motivated and is unlikely to provide a robust scaling with changing atmospheric parameters. An improved 1D convection description including non-local effects like overshooting is clearly desirable to handle this situation. An empirical calibration of $\alpha_{\mathrm{MLT}}$ using giants is likely to suffer from ambiguities related to the way turbulent pressure is treated in $1 \mathrm{D}$ models.

\section{Modelling the photometric micro-variability in HD 49933}

The stochastically changing granular flow pattern on the surface of late-type stars causes low-level residual brightness fluctuations in stellar disk-integrated light. Ludwig (2006) described a method to obtain predictions of the observable temporal power spectrum from local-box RHD simulations if - besides the atmospheric parameters - the radius of the target star is known. One of the first primary asteroseismic targets of 


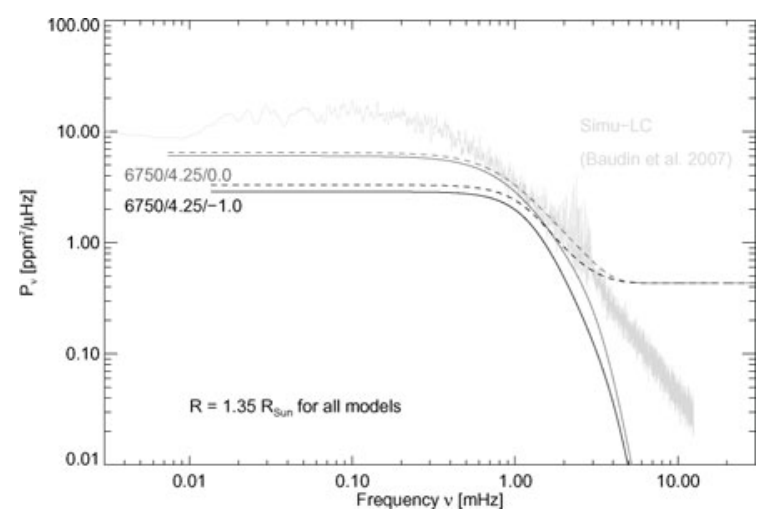

Figure 5. Predictions for the temporal power spectral density of the photometric brightness fluctuations exhibited by HD 49933. 3D RHD simulations are depicted by solid lines and labelled by $T_{\text {eff }} / \log g /[\mathrm{M} / \mathrm{H}]$. Dashed lines depict the result when adding the estimated photometric noise level. For further comparison a spectrum obtained with the COROT light curve simulator (Baudin et al. 2007) is shown.

the COROT satellite mission was the metal-depleted $(-0.3 \geqslant[\mathrm{M} / \mathrm{H}] \geqslant-0.4)$ F-dwarf HD 49933 for which a high-precision photometric light-curve was obtained. The data is not released yet so that we are only showing theoretical predictions obtained from two RHD simulation runs at $T_{\text {eff }}=6750 \mathrm{~K}, \log g=4.25$ and two metalicities $[\mathrm{M} / \mathrm{H}]=0.0$ and -1.0 bracketing the observed metalicity of HD 49933 and having atmospheric parameters close to the spectroscopically measured values. Figure 5 depicts the power spectra obtained from the two RHD simulations. For smoothing, the raw RHD spectra were fitted by a simple analytical model, and oscillatory peaks from the acoustic eigenmodes of the computational box were removed. Hence, the pure convection-related signal is shown. The observed signal should fall between the two model predictions, at least at around $1 \mathrm{mHz}$ where the photon noise and the signal due to magnetic activity are expected not to dominate. Unfortunately, at present it looks that this is not quite the case. An actual comparison will be presented in an upcoming paper by Ludwig \& Samadi, so stay tuned.

\section{References}

Böhm-Vitense, E. 1958, Zs. f. Astrophys. 46, 108

Baudin, F., Samadi, R., Appourchaux, T., \& Michel, E. 2007, arXiv:0710.3378

Christensen-Dalsgaard J. 1997, in: Pijpers, F. P. and Christensen-Dalsgaard, J. and Rosenthal, C. S. (eds.), SCORe'96 : Solar Convection and Oscillations and their Relationship, ASSL 225, 3

Freytag, B., Ludwig, H.-G., \& Steffen, M. 1999, in: Gimenez, A. and Guinan, E. F. and Montesinos, B. (eds.), Theory and Tests of Convection in Stellar Structure, ASPC 173, 225

Lebreton Y., Fernandes J., \& Lejeune T. 2001, A\& $A$ 374, 540

Ludwig, H.-G., Freytag, B., Steffen, M. 1999, A\&A $A 46,111$

Ludwig, H.-G. 2006, A $B A$ 445, 661

Mihalas, D. 1978, Stellar Atmospheres, Freeman and Company

Trampedach, R. 2007, in: Unsolved Problems in Stellar Physics: A Conference in Honour of Douglas Gough, AIPCS 948, 141

\section{Discussion}

Canuto: Do you really believe that $\alpha>1$ is physically realistic?

LUDWIG: Yes, I do not see a problem with $\alpha>1$ in the framework of standard mixinglength theory (MLT). On the one hand, MLT is a simplistic approach so that its intrinsic 
parameters should not interpret overly strictly. On the other hand, the velocity field in stratified convection shows a correlation length which regularly supersedes the local pressure scale height (see, e.g., the works of Chan \& Sofia 1987, 1989).

Turck-CHIEzE: (1) Do you apply your 3D atmospheric model to predict the solar granulation in comparing with the GOLF/SoHO spectrum working in Doppler velocity? (2) Don't you think that the slope at low frequency of the CoRoT HD 49933 comes from an instrumental effect (naturally increase at low freq in $1 / \mathrm{f}$ ).

LUDWIG: (1) I did not apply the 3D atmosphere model yet to obtain predictions for the granulation signal in Doppler signal. The scaling procedure from local box to disk - integrated signal has not been fully worked out yet. Moreover, if one intends to do a detailed modeling of the involved spectral line(s) the necessary computational effort becomes substantial. (2) No, not in the frequency range $>0.01 \mathrm{mHz}$. Calibration stars have been observed with CoRoT which do not show this increase which makes the interpretation as signature of magnetic activity much more likely.

Christensen-Dalsgaard: Comment: In parallel region, Tranmpedach has developed determination of $\alpha_{M L T}$ from Nordlund-Stein simulations, going back to $\sim 1996$. It would be very interesting to compare these two sets of results.

LuDwiG: Yes, Regner Trampedach presented values of the mixing-length parameter obtained from a small number $(\sim 6) 3 \mathrm{D}$ models at this time. The qualitative functional dependence of the ML parameter agreed with the dependence found in the 2D models, despite differences in the numerical approaches. It would be certainly worthwhile to take a fresh look at the predictions of the two sets of 3D models especially based on the analogous sets of 1D comparison models. 